\title{
A Network Pharmacology-Based Approach for Exploring Key Active Compounds and Pharmacological Mechanisms of Tangshen Formula for Treatment of Diabetic Nephropathy
}

\author{
Weie Zhou $\mathbb{D}^{1,2,3}$ Xuefeng Zhou, ${ }^{1}$ Yuan Zhang, ${ }^{3}$ Yuyang Wang $\mathbb{D}^{1},{ }^{1}$ Wenjie $W u,{ }^{3}$ \\ Haojun Zhang, ${ }^{1}$ Tingting Zhao, ${ }^{1}$ Liang Peng, ${ }^{1}$ Hailing Zhao, ${ }^{1}$ and Ping Li ${ }^{1,2}$ \\ ${ }^{1}$ Beijing Key Laboratory for Immune-Mediated Inflammatory Diseases, Department of Nephrology, China- \\ Japan Friendship Hospital, Beijing 100029, China \\ ${ }^{2}$ Graduate School of Peking Union Medical College, Chinese Academy of Medical Sciences and Peking Union Medical College, \\ Beijing 100730, China \\ ${ }^{3}$ Institute of Food Safety, Chinese Academy of Inspection \& Quarantine, 100176 Beijing, China
}

Correspondence should be addressed to Ping Li; 1p8675@163.com

Received 12 August 2020; Revised 6 January 2021; Accepted 8 February 2021; Published 15 February 2021

Academic Editor: Hiroshi Okamoto

Copyright (c) 2021 Weie Zhou et al. This is an open access article distributed under the Creative Commons Attribution License, which permits unrestricted use, distribution, and reproduction in any medium, provided the original work is properly cited.

Diabetic nephropathy (DN) is one of the common and severe microvascular complications of diabetes mellitus (DM). The occurrence and development of DN are related to multiple factors in the human body, which makes DN a complex disease, and the pathogeneses of DN have not yet been fully illustrated. Furthermore, DN lacks effective drugs for treatment nowadays. Chinese herbal medicine ( $\mathrm{CHM}$ ) often shows the feature of multicomponents, multitargets, multipathways, and synergistic effects and shows a promising source of new therapeutic drugs for DN. As a CHM, Tangshen Formula (TSF) was used to treat DN patients in China. However, its bioactive compounds and holistic pharmacological mechanisms on DN are both unclear. A network pharmacology approach was firstly applied to explore multiple active compounds and multiple key pharmacological mechanisms for TSF treating DN by drug-targeted interaction databases, herb-compound-target network, protein-protein interaction network, compound-target-pathway network, and analysis methods. And the results showed that TSF have the characteristic of multicomponents, multitargets, multipathways, and synergistic effects for treating DN. The quercetin, naringenin, kaempferol, and isorhamnetin as key active compounds and the PI3K-Akt signaling pathway, TNF signaling pathway, nonalcoholic fatty liver disease (NAFLD), focal adhesion, rap1 signaling pathway, T cell receptor signaling pathway, MAPK signaling pathway, and insulin resistance as the key molecular mechanisms play important roles in TSF treating DN. Moreover, quercetin, naringenin, kaempferol, and isorhamnetin were successfully detected in TSF by the UHPLC-MS/MS analysis method. And their concentrations were $0.224,8.295,0.0564$, and $0.0879 \mathrm{mg} \cdot \mathrm{kg}^{-1}$, respectively. The present findings not only provide new insights for a deeper understanding of the constituent basis and pharmacology of TSF but also provide guidance for further pharmacological studies on TSF.

\section{Introduction}

Diabetic nephropathy (DN) is one of the common and severe microvascular complications of diabetes mellitus (DM) and the major substantial cause of end-stage renal disease (ESRD) which contributes to morbidity and mortality. According to the epidemiological evidence, about $15-40 \%$ of diabetic patients may deteriorate to $\mathrm{DN}[1,2]$. The incidence rate of
DN has become a global health problem and needs to be early examined and treated to delay progression and to prevent adverse outcomes. The occurrence and development of DN are related to the multiple factors in the human body, which makes DN a complex disease, and the pathogeneses of DN have not yet been fully illustrated. Currently, as we know, the main clinical characteristic of DN is to increase glomerular filtration ratio (GFR), proteinuria, and creatinine levels in 
the initial state and decrease GFR in the end stage [3]. And the major pathological change of $\mathrm{DN}$ included glomerular hypertrophy which thickened the extracellular matrix and incrassated the glomerular basement membrane (GBM), hyperplastic mesangial matrix, and so on [4]. And they eventually contributed to produce glomerular sclerosis and renal interstitial fibrosis [4]. Current researches believe that the occurrence and development of $\mathrm{DN}$ are mainly related to metabolic disorders (abnormal lipid metabolism, glycometabolism disorder, etc.), protein kinase $\mathrm{C}$ activation, oxidative stress, inflammatory response, hemodynamic changes, and genetic factors [5]. At present, the standard agents involved drugs controlling hyperglycemia and high blood pressure such as angiotensin converting enzyme inhibitors or angiotensin receptor antagonist antiagents (ACEI/ARB), and renin angiotensin aldosterone system blockade and sodium glucose cotransporter 2 (SGLT2) inhibition are often used for the treatment of DN according to its pathological factors [6, 7]. However, these drugs cannot prevent the DM patients continuing to develop DN leading to glomerulosclerosis, tubulointerstitial fibrosis, and even cardiovascular comorbidities, because these drugs often tend to act on a single target or sole molecular mechanism. Therefore, multicompound drugs which act on multiple targets and multipathways are desperately required for the treatment of $\mathrm{DN}$.

The Chinese herbal medicine (CHM) is often characteristic of multicomponents, multitargets, multipathways, and synergistic effects and shows a promising source of new therapeutic drugs for DN [8]. Tangshen Formula (TSF) is formulated based on the individual property of $\mathrm{CHM}$ for treating DN. Based on previous works, TSF significantly alleviated proteinuria $[9,10]$, enhanced estimated glomerular filtration rate (eGFR) [9], and improved phospholipid metabolism [11] in DN patients. Moreover, TSF was demonstrated to inhibit kidney lipogenesis and increase kidney fatty acid oxidation though AMPK-PPAR $\alpha$ in $\mathrm{db} / \mathrm{db}$ mice [12], improving renal inflammation via blockade of NF- $\kappa \mathrm{B}$ [13], ameliorating renal fibrosis by TGF- $\beta / \operatorname{Smad} 3$ [13], improving phospholipid metabolism by inhibiting the protein kinase $\mathrm{C}$ (PKC) pathway, and reducing the phospholipase A2 activity [11]. However, its bioactive compounds are still unclear. Moreover, their holistic and key pharmacological mechanisms on $\mathrm{DN}$ are also unclear although some sole pharmacological mechanisms had been explored for TSF. Network pharmacology was proposed to investigate synergistic effects and potential mechanisms of multiple compounds through the multilayered networks by Hopkins in 2008 [14], and it is a suitable means to explore bioactive compounds and holistic molecular mechanism of CHM.

This study is aimed at exploring key active compounds and pharmacological mechanisms of TSF for treatment of DN by network pharmacology. The technical route of this study is illustrated in Figure 1. In this study, we would firstly explore the bioactive compounds and holistic potential mechanism of TSF on DN by drug-targeted interaction databases, herb-compound-target network analysis, protein-protein interaction network compound-targetpathway network analysis, and quantitative analytical method.

\section{Materials and Methods}

2.1. Screening Active Compounds and Their Potential Targets for TSF. TSF contains seven medicinal materials that consist of Astragalus membranaceus (AM), Cornus officinalis (CO), Rehmannia glutinosa (RG), Rheum palmatum (RP), Panax notoginseng (PNG), Euonymus alatus (EA), and Citrus aurantium (CA). All their chemical compounds were collected by Traditional Chinese Medicine System Pharmacology (TCMSP) and analysis platform database (http://lsp.nwu .edu.cn/tcmsp.php) which is a system pharmacology platform that can show the relationships between herbal ingredients, targets, and diseases. It can provide $502 \mathrm{CHM}$ registered in Chinese pharmacopoeia and 13,729 ingredients from scientific publications and literatures. Thus, it is enough for TSF searching its ingredients. Moreover, it also can provide a bioactive ingredients screening process based on absorption, distribution, metabolism, and excretion (ADME) features which contribute to the therapeutic effects of the drug. And oral bioavailability (OB) is a good indicator of pharmacokinetic parameter for screening candidate active compounds which is efficient in oral administration for drug delivery into systemic circulation. In addition, drug-likeness (DL) often describes the pharmacokinetic and pharmaceutical properties of compounds, such as solubility and potency, and is often used to evaluate a compound that has the potential to be further developed into drugs. In order to acquire ingredients with good oral absorption, usage, and biological properties, the active candidate compounds of TSF were screened under the conditions of $\mathrm{OB} \geq 30 \%$ and $\mathrm{DL} \geq 0.18$.

For each candidate active compound, their corresponding putative targets were gathered from TCMSP and literatures in PubChem (https://pubchem.ncbi.nlm.nih.gov/) and predicted by the STITCH database (http://stitch.embl .de/) under the condition of Homo sapiens. It is worth noting that STITCH would identify similar chemical molecules with a similarity score greater than 0.99 to ensure the reliability of the collected target for the compounds which cannot find their targets in TCMSP. In addition, the targets would also be obtained from literatures in the PubChem database for these compounds whose targets cannot be found in TCMSP. And the UniProt database (https://www.uniprot.org/) was utilized to standardize target gene names.

2.2. Screening Potential Targets for DN. The DN-related targets were acquired from two sources: DisGeNET (http:// www.disgenet.org/) and GeneCards (https://www.genecards .org/, ver. 49.0). The DisGeNET database is one of the largest publicly available platforms of gene collection which is integrated with multifunction data including various classes of genetic disease, human associated genes, and experimental research. The GeneCards database is one of the comprehensive multifunctional online catalog platforms for linkage among genomics, proteomics, transcriptomics, and disease. Thus, the keywords "Diabetic Nephropathy" were used to retrieval all targets related to $\mathrm{DN}$ in the two databases under the condition of Homo sapiens. Furthermore, TSF-DN cotargets were intersected by the OmicShare platform (https:// 


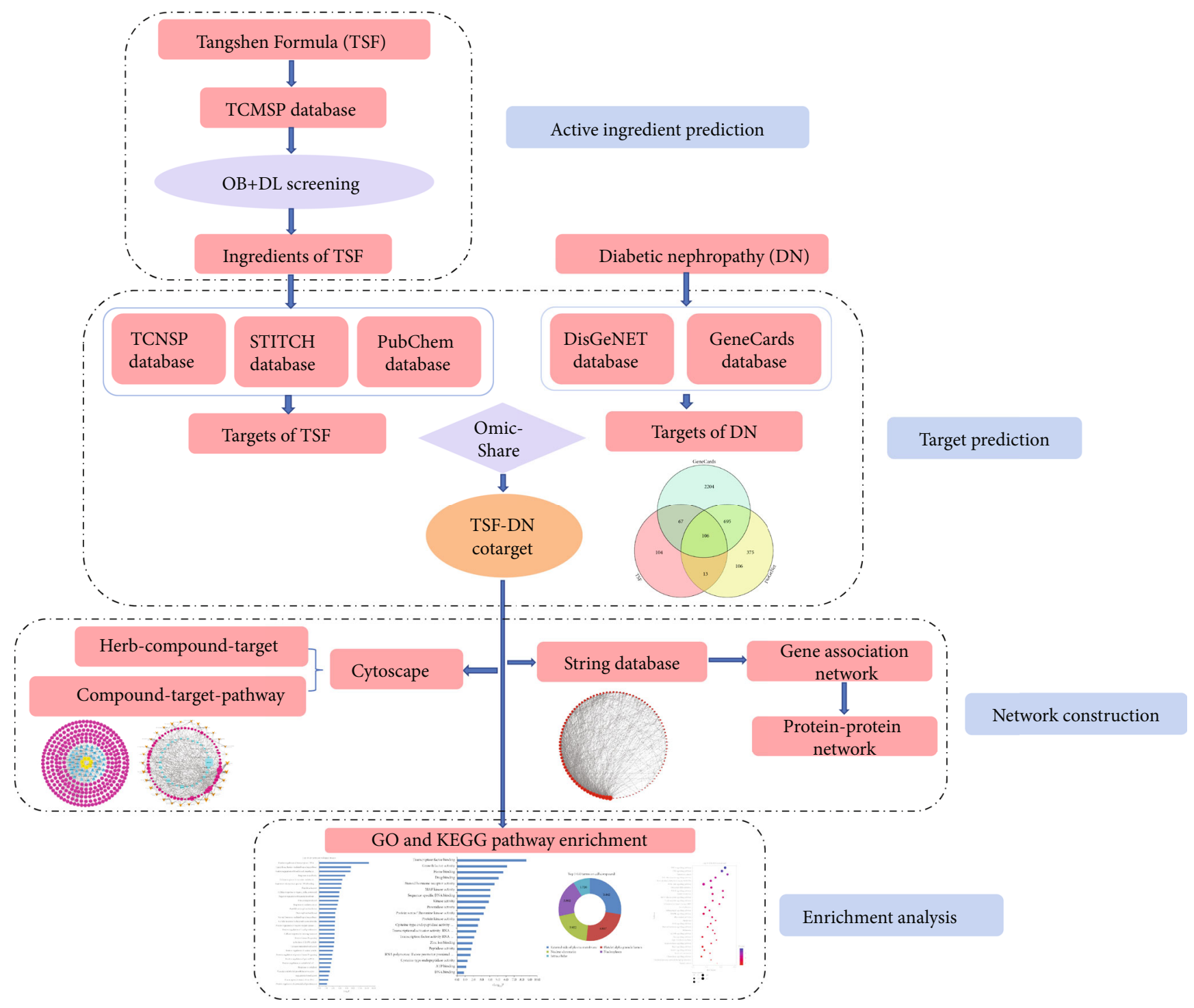

Figure 1: The whole framework based on network pharmacology for deciphering active ingredients and pharmacological mechanism of Tangshen Formula (TSF) acting on diabetic nephropathy.

www.omicshare.com/) and taken as potential targets for the compounds of TSF treating DN.

\subsection{Gene Association Interaction Data of TSF-DN Cotargets.} The putative TSF target-therapeutic target of DN interactions was structured based on the STRING database (https://string-db.org/) which can provide almost all functional interactions including coexpression, physical neighborhood, gene fusion, and cooccurrence between the expressed proteins. Moreover, the gene association information is often from literature mining, experiments, and computation-based prediction. In order to obtain key TSF$\mathrm{DN}$ cotarget interactions, a high confidence score was set to $\geq 0.700$ under the condition of "Homo sapiens."

2.4. Gene Ontology (GO) and Kyoto Encyclopedia of Genes and Genomes (KEGG) Pathway Enrichment Analyses. In order to clarify biological process (BP), molecular function
(MF), and cell component (CC) for TSF on DN, the TSFDN cotargets were utilized for GO enrichment analysis by Database Visualization and Integrated Discovery software (DAVID software, https://david.ncifcrf.gov/). And the key terms for GO on BP, MF, and CC were collected from the upper $50 \%$ cluster terms under the condition of $P$ value less than 0.05. Furthermore, a pathway enrichment analysis involved in putative TSF-DN cotargets was also employed by DAVID which is the pathway data obtained from the KEGG database (http://www.genome.jp/kegg/), and the key pathway was obtained under the condition of $P$ value less than 0.001 .

2.5. Network Construction and Analysis. As we know, the degree, betweenness centrality, and closeness centrality parameters are three important topological features. And the nodes with the three topological features higher than 
TABLE 1: Basic information of 50 candidate active ingredients was collected for the Tangshen Formula (TSF).

\begin{tabular}{|c|c|c|c|c|c|}
\hline No. & TCMSP ID & Molecule name & $\begin{array}{l}\text { Molecular } \\
\text { weight }\end{array}$ & OB & DL \\
\hline 1 & MOL000033 & $\begin{array}{l}\text { (3S,8S,9S,10R,13R,14S,17R)-10,13-Dimethyl-17-[(2R,5S)-5-propan-2-yloctan-2-yl]- } \\
\text { 2,3,4,7,8,9,11,12,14,15,16,17-dodecahydro-1H-cyclopenta[a]phenanthren-3-ol }\end{array}$ & 428.82 & 36.23 & 0.78 \\
\hline 2 & MOL000096 & $(-)$-Catechin & 290.29 & 49.68 & 0.24 \\
\hline 3 & MOL000098 & Quercetin & 302.25 & 46.43 & 0.28 \\
\hline 4 & MOL000211 & Mairin & 456.78 & 55.38 & 0.78 \\
\hline 5 & MOL000239 & Jaranol & 314.31 & 50.83 & 0.29 \\
\hline 6 & MOL000296 & Hederagenin & 414.79 & 36.91 & 0.75 \\
\hline 7 & MOL000354 & Isorhamnetin & 316.28 & 49.6 & 0.31 \\
\hline 8 & MOL000358 & Beta-sitosterol & 414.79 & 36.91 & 0.75 \\
\hline 9 & MOL000359 & Sitosterol & 414.79 & 36.91 & 0.75 \\
\hline 10 & MOL000371 & 3,9-di-O-Methylnissolin & 314.36 & 53.74 & 0.48 \\
\hline 11 & MOL000378 & 7-O-Methylisomucronulatol & 316.38 & 74.69 & 0.3 \\
\hline 12 & MOL000379 & 9,10-Dimethoxypterocarpan-3-O- $\beta$-D-glucoside & 462.49 & 36.74 & 0.92 \\
\hline 13 & MOL000380 & (6aR,11aR)-9,10-Dimethoxy-6a,11a-dihydro-6H-benzofurano[3,2-c]chromen-3-ol & 300.33 & 64.26 & 0.42 \\
\hline 14 & MOL000387 & Bifendate & 418.38 & 31.1 & 0.67 \\
\hline 15 & MOL000392 & Formononetin & 268.28 & 69.67 & 0.21 \\
\hline 16 & MOL000398 & Isoflavanone & 316.33 & 109.99 & 0.3 \\
\hline 17 & MOL000417 & Calycosin & 284.28 & 47.75 & 0.24 \\
\hline 18 & MOL000422 & Kaempferol & 286.25 & 41.88 & 0.24 \\
\hline 19 & MOL000433 & FA & 441.45 & 68.96 & 0.71 \\
\hline 20 & MOL000442 & 1,7-Dihydroxy-3,9-dimethoxy pterocarpene & 314.31 & 39.05 & 0.48 \\
\hline 21 & MOL000449 & Stigmasterol & 412.77 & 43.83 & 0.76 \\
\hline 22 & MOL000471 & Aloe-emodin & 270.25 & 83.38 & 0.24 \\
\hline 23 & MOL001040 & (2R)-5,7-Dihydroxy-2-(4-hydroxyphenyl)chroman-4-one & 272.27 & 42.36 & 0.21 \\
\hline 24 & MOL001420 & ZINC04073977 & 412.77 & 38 & 0.76 \\
\hline 25 & MOL001494 & Mandenol & 308.56 & 42 & 0.19 \\
\hline 26 & MOL001495 & Ethyl linolenate & 306.54 & 46.1 & 0.2 \\
\hline 27 & MOL001755 & 24-Ethylcholest-4-en-3-one & 412.77 & 36.08 & 0.76 \\
\hline 28 & MOL001771 & Poriferast-5-en-3beta-ol & 414.79 & 36.91 & 0.75 \\
\hline 29 & MOL001792 & DFV & 256.27 & 2.57 & 32.76 \\
\hline 30 & MOL002235 & EUPATIN & 360.34 & 50.8 & 0.41 \\
\hline 31 & MOL002268 & Rhein & 284.23 & 47.07 & 0.28 \\
\hline 32 & MOL002281 & Toralactone & 272.27 & 46.46 & 0.24 \\
\hline 33 & MOL002297 & Daucosterol_qt & 386.73 & 35.89 & 0.7 \\
\hline 34 & MOL002341 & Hesperetin & 302.3 & 70.31 & 0.27 \\
\hline 35 & MOL002879 & Diop & 390.62 & 43.59 & 0.39 \\
\hline 36 & MOL002883 & Ethyl oleate (NF) & 310.58 & 32.4 & 0.19 \\
\hline 37 & MOL003137 & Leucanthoside & 462.44 & 32.12 & 0.78 \\
\hline 38 & MOL004328 & Naringenin & 272.27 & 59.29 & 0.21 \\
\hline 39 & MOL005100 & 5,7-Dihydroxy-2-(3-hydroxy-4-methoxyphenyl)chroman-4-one & 302.3 & 47.74 & 0.27 \\
\hline 40 & MOL005344 & Ginsenoside rh2 & 622.98 & 36.32 & 0.56 \\
\hline 41 & MOL005481 & 2,6,10,14,18-Pentamethylicosa-2,6,10,14,18-pentaene & 342.67 & 33.4 & 0.24 \\
\hline 42 & MOL005486 & 3,4-Dehydrolycopen-16-al & 548.92 & 46.64 & 0.49 \\
\hline 43 & MOL005503 & Cornudentanone & 378.56 & 39.66 & 0.33 \\
\hline 44 & MOL005530 & Hydroxygenkwanin & 300.28 & 36.47 & 0.27 \\
\hline 45 & MOL005531 & Telocinobufagin & 402.58 & 69.99 & 0.79 \\
\hline 46 & MOL005552 & Gemin D & 634.49 & 68.83 & 0.56 \\
\hline 47 & MOL005828 & Nobiletin & 402.43 & 61.67 & 0.52 \\
\hline
\end{tabular}


TABLE 1: Continued.

\begin{tabular}{|c|c|c|c|c|c|}
\hline No. & TCMSP ID & Molecule name & $\begin{array}{c}\text { Molecular } \\
\text { weight }\end{array}$ & OB & DL \\
\hline 48 & MOL007475 & Ginsenoside f2 & 785.14 & 36.43 & 0.25 \\
\hline 49 & MOL008457 & Tetrahydroalstonine & 352.47 & 32.42 & 0.81 \\
\hline 50 & MOL013381 & Marmin & 332.43 & 38.23 & 0.31 \\
\hline
\end{tabular}

Note: OB: oral bioavailability; DL: drug-likeness.

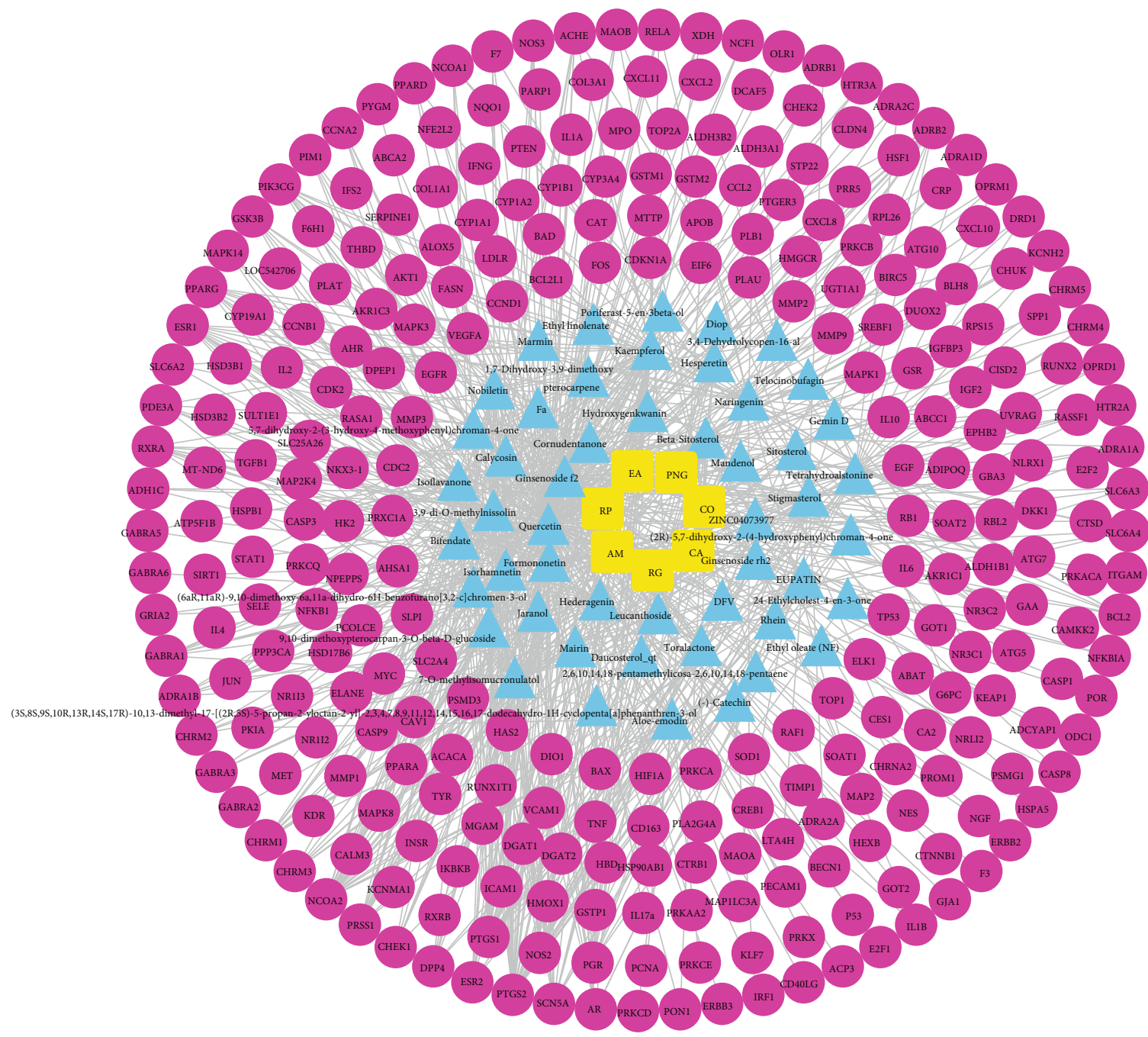

FIGURE 2: The herb-compound-target network was conducted for Tangshen Formula (TSF). The yellow rectangle nodes represent herbs (AM: Astragalus membranaceus; CO: Cornus officinalis; RG: Rehmannia glutinosa; RP: Rheum palmatum; PNG: Panax notoginseng; EA: Euonymus alatus, CA: Citrus aurantium). The blue triangle nodes represent compounds, the red circle nodes represent the potential targets, and the lines among inner nodes display the relationship among herbs, compounds, and targets.

the corresponding median values can be used as the major hubs.

The herb-component-target network was analyzed by Cytoscape software (https://cytoscape.org/, version 3.8.0) to reflect visual and reliable relationship among the herbs, active compounds, and potential targets for TSF. According to this network, the key active ingredients can be predicted for TSF with the three topological features including the degree, betweenness centrality, and closeness centrality which were higher than the corresponding median values of all the nodes in the network.

Protein-protein interactional network were analyzed by Cytoscape software. And the analyzed data from the STRING database were input to Cytoscape software for drawing and analyzing the TSF target-DN target network. Therefore, the key TSF targets treated on DN were screening with the three 


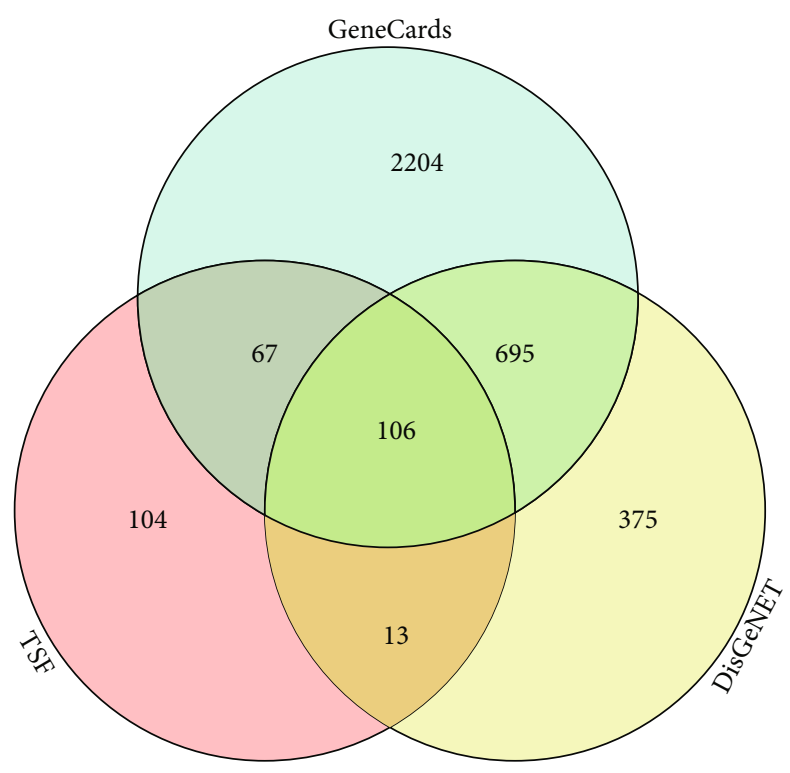

FIGURE 3: Intersection of target genes were mapped between diabetic nephropathy (DN) and Tangshen Formula (TSF).

topological features higher than the corresponding median values in a network. According to the result, the key targets' corresponding compounds were obtained.

The ingredient-target-pathway network was analyzed by Cytoscape software using compounds, cotargets, and KEGG pathways. And the key pharmacological mechanisms were obtained for TSF on treatment of DN with the three topological features higher than the corresponding median values.

2.6. UHPLC-MS/MS Analytical Method for 4 Key Ingredients in TSF. The quantitative analytical method for key ingredients was carried out on a UHPLC-MS/MS apparatus. The model of mass spectrometer (MS) consisted of a Qtrap 6500 (Applied Biosystems, NY, USA) with an electrospray ionization (ESI) source for molecule ionization. The optimized ESI temperature was set at $450^{\circ} \mathrm{C}$ and ion spray voltage at $-4500 \mathrm{~V}$ for negative mode. The curtain gas was $35 \mathrm{psi}$ and collision gas pressure was medium. Ion source gas 1 was 40 and ion source gas 2 was 45 . The quantitative data in this study were acquired using MRM mode. A UHPLC model 30A (Shimadzu, Japanese) consisted of a binary pump, degasser, and autosampler. The peak areas of each constituent were automatically integrated using Analyst software. The separation condition was carried out by ACQUITY UPLC HSS T3 Column $(2.1 \mathrm{~mm} \times 100 \mathrm{~mm}, 1.8 \mu \mathrm{m}$, Waters $)$. The mobile phase consisted of acetonitrile (solvent A) and aqueous solution (solvent $\mathrm{B}$ ). The optimized gradient elution conditions were used as follows: $10 \% \mathrm{~A}$ at $0-1.0 \mathrm{~min}, 10-35 \%$ $\mathrm{A}$ at $1.0-2.0 \mathrm{~min}, 35-70 \% \mathrm{~A}$ at $2.0-5.0 \mathrm{~min}, 70-98 \% \mathrm{~A}$ at $5.0-$ $6.0 \mathrm{~min}, 98-10 \% \mathrm{~A}$ at $6.0-6.5 \mathrm{~min}$, and $10-10 \% \mathrm{~A}$ at $6.5-$ $10.0 \mathrm{~min}$. The flow rate was $0.3 \mathrm{~mL} / \mathrm{min}$, and the injection volume was $5.0 \mu \mathrm{L}$, and the column temperature was $30^{\circ} \mathrm{C}$.

Quercetin (ID number: 4CXN-LCMK, purity: $97.4 \%$ ) and kaempferol (ID number: HBSF-75L8, purity: 93.2\%) were purchased from China Food and Drug Testing Institute (Beijing, China). Mass grade acetonitrile and metha- nol were purchased from Fisher Scientific Inc. (Waltham, MA, US). Deionized water was purified using a Milli-Q water-purification system (Millipore, Bedford, USA). Naringenin (Lot number: A0660020, purity: 99.6\%) and isorhamnetin (Lot number: U2050025, purity: 99.4\%) were purchased from ANPEL laboratory Technologies Inc. (Shanghai, China).

Individual stock solution of reference substances quercetin, naringenin, kaempferol, and isorhamnetin were, respectively, prepared in methanol at a concentration of $1 \mathrm{mg} / \mathrm{mL}$. Then, all stock solutions were stored at $-20^{\circ} \mathrm{C}$ until use. A series of mixed standard solutions were prepared to the desired concentration $(0.01,0.02,0.05,0.1,0.2,0.5,1,2,5$, $10,20,50$, and $100 \mathrm{ng} / \mathrm{mL}$ ) in methanol by diluting stock solution.

For a sample solution, $50 \mathrm{mg}$ TSF was accurately weighted into a centrifuge tube and extracted with $10 \mathrm{~mL}$ of $50 \%$ methanol aqueous solution by vortexing in $5 \mathrm{~min}$ and ultrasonicating in $20 \mathrm{~min}$. Then, the extracted solution was filtered through a syringe filter $(0.22 \mu \mathrm{m})$ for further UHPLC-MS/MS analysis.

\section{Results}

3.1. Candidate Active Compounds and Their Potential Targets for TSF. In this study, the available ingredients including 20 compounds in Astragalus membranaceus (AM), 20 compounds in Cornus officinalis (CO), 2 compounds in Rehmannia glutinosa (RG), 16 compounds in Rheum palmatum (RP), 8 compounds in Panax notoginseng (PNG), 8 compounds in Euonymus alatus (EA), and 5 compounds in Citrus aurantium (CA) were obtained under the screening condition of oral bioavailability $(\mathrm{OB}) \geq 30 \%$ and drug - likeness $(\mathrm{DL}) \geq 0.18$ properties. After getting rid of repetitive and other invalid ingredients, 50 candidate active compounds were selected out for TSF (Table 1). Finally, 290 potential targets were identified for 50 candidate active compounds in TSF after removing the duplicate ones (Supplementary Table S1).

3.2. Herb-Compound-Target Network Analysis for TSF. To further understand the interaction relationship among the herb, compounds, and their corresponding targets for TSF, an herb-compound-target network was constructed for TSF by mapping 7 herbs to 50 compounds linked to 290 potential targets, as shown in Figure 2. Based on the network analysis, 349 nodes and 786 edges were obtained from this network (Supplementary Table S2-S3). And AM has the highest degree, betweenness centrality, and closeness centrality among 7 herbs and indicated its effect as emperor drug in TSF. Furthermore, quercetin, kaempferol, ginsenoside f2, 7O-methylisomucronulatol, beta-sitosterol, naringenin, formononetin, isorhamnetin, stigmasterol, nobiletin, and isoflavanone had higher degree, betweenness centrality, and closeness centrality than the corresponding median values among 50 candidate active ingredients. The results suggested that these 11 components probably served as significant therapeutic compounds in TSF. 


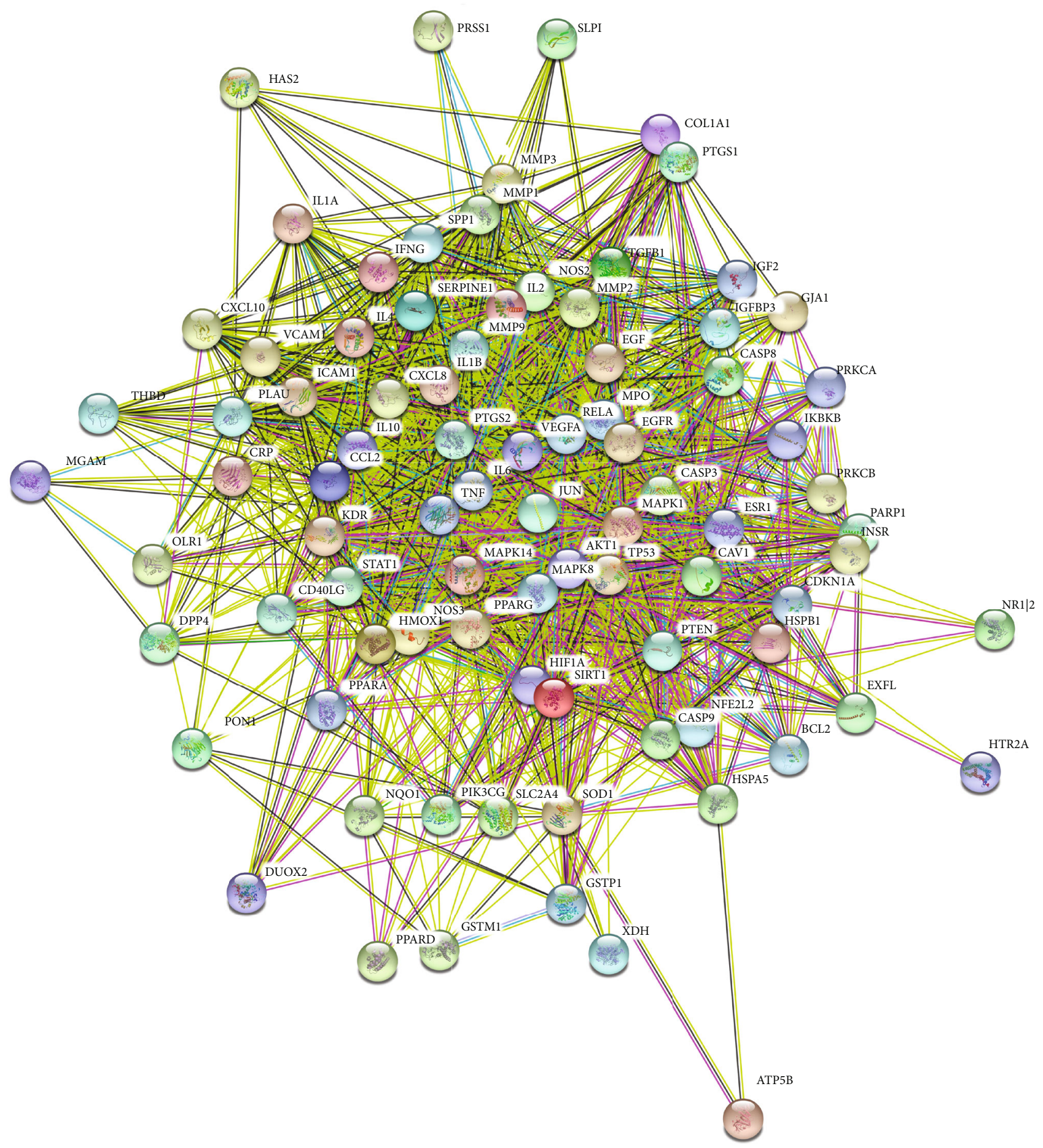

FIgURE 4: Common target gene association interactions network between TSF and DN from the STRING database. Each bubble node represents a target, and the 3D structure in the bubble nodes represent that the protein spatial structure is known or predicted. The lines among inner nodes display the relationship between different targets, and the width of lines was based on the strength of data support.

3.3. TSF-DN Cotarget Analysis. In order to obtain the TSF targets associated with DN, the TSF-DN cotargets were intersected and shown as a Venn diagram in Figure 3 and Supplementary Table S4. And the 106 TSF-DN cotargets were imputed to the STRING database for analysis. From the STRING result data, a total of 100 nodes and 878 edges were acquired, and the average combined score of the edges is 0.864 . The nodes indicated the TSF-DN cotargets and the edges represent the coexpression, physical neighborhood, gene fusion, and cooccurrence between a pair of action targets, as shown in Figure 4. And the combined score shows their high action intensity with high scores. The detail gene association network map with above 0.7 combined scores are shown in Supplementary Table S5. 


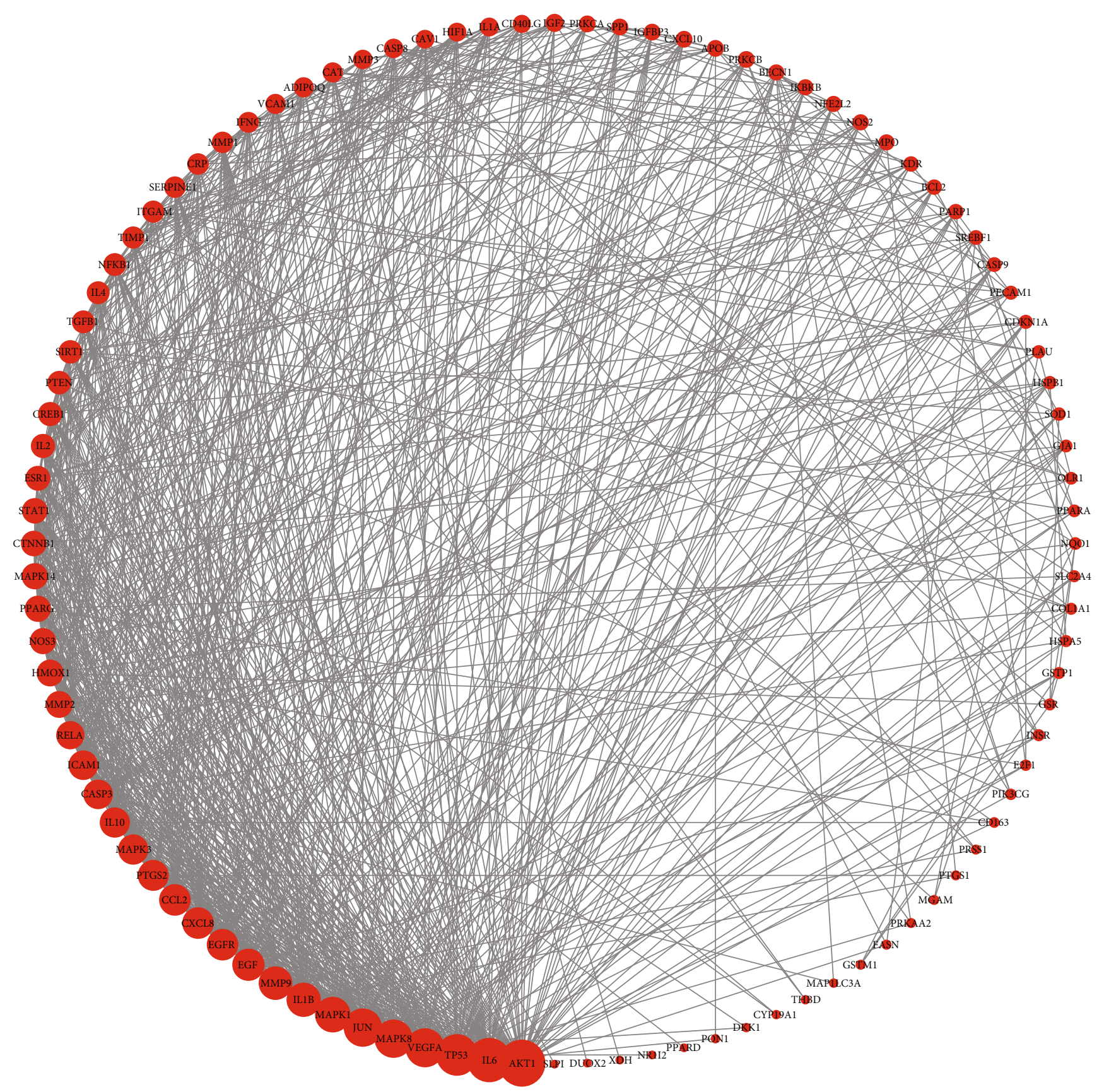

FIGURE 5: Protein-protein interaction networks were analyzed by Cytoscape software. The red circle represents 100 potential targets from the STRING data, the size of circle represents the degree, and the lines among inner nodes display the relationship between different targets.

3.4. Protein-Protein Interaction Network for Key Target Associated with TSF Treating DN. Protein-protein interaction network whose targets were from the STRING data were further drawn and analyzed by Cytoscape software. The network was shown in Figure 5. And the 27 key potential targets for TSF associated with DN with the three topological features greater than the corresponding median values are gathered in Table 2. In addition, the corresponding ingredients acting on the 27 key potential targets can be correspondingly obtained. The results indicated that many key active compounds such as quercetin, kaempferol, ginsenoside f2, 7-Omethylisomucronulatol, beta-sitosterol, naringenin, formo- nonetin, isorhamnetin, stigmasterol, nobiletin, and isoflavanone in TSF were associated with DN targets.

3.5. Gene Function Analysis. The 106 TSF-DN cotarget genes were imported into the DAVID system for Gene Ontology (GO) cluster analysis. The 26 cluster terms whose enrichment scores were ranged from 1.257 to 6.384 were collected. And the $50 \%$ cluster terms whose enrichment scores were up to 3.244 were obtained. Furthermore, the top 30 remarkably enriched biological process (BP) terms, the top 20 remarkably enriched molecular function (MF) terms, and the top 5 remarkably enriched cell compound (CC) terms from the 
TABLE 2: The network topology parameters and active compounds were collected for 27 key TSF-DN cotargets from protein-protein international network.

\begin{tabular}{|c|c|c|c|c|c|}
\hline No. & Name & Degree & $\begin{array}{c}\text { Betweenness } \\
\text { centrality }\end{array}$ & $\begin{array}{l}\text { Closeness } \\
\text { centrality }\end{array}$ & DN target corresponding compounds of TSF \\
\hline 1 & AKT1 & 58 & 0.113 & 0.697 & Quercetin, kaempferol, naringenin \\
\hline 2 & TP53 & 50 & 0.093 & 0.656 & Quercetin, aloe-emodin, nobiletin \\
\hline 3 & IL6 & 54 & 0.077 & 0.678 & Quercetin \\
\hline 4 & CAT & 18 & 0.050 & 0.521 & Naringenin, ginsenoside $\mathrm{f} 2$ \\
\hline 5 & MAPK8 & 45 & 0.049 & 0.639 & Kaempferol, nobiletin \\
\hline 6 & VEGFA & 46 & 0.047 & 0.631 & Quercetin \\
\hline 7 & JUN & 45 & 0.037 & 0.631 & Quercetin, kaempferol, beta-sitosterol, rhein, nobiletin \\
\hline 8 & CASP3 & 32 & 0.037 & 0.579 & Quercetin, kaempferol, beta-sitosterol, aloe-emodin, ginsenoside rh2 \\
\hline 9 & CTNNB1 & 26 & 0.034 & 0.544 & Ginsenoside f2 \\
\hline 10 & MMP9 & 38 & 0.031 & 0.596 & Quercetin \\
\hline 11 & HMOX1 & 28 & 0.028 & 0.569 & Quercetin, kaempferol \\
\hline 12 & MAPK1 & 41 & 0.028 & 0.611 & Quercetin, naringenin \\
\hline 13 & NFKB1 & 22 & 0.026 & 0.538 & Isoflavanone \\
\hline 14 & EGFR & 35 & 0.024 & 0.589 & Quercetin \\
\hline 15 & EGF & 36 & 0.024 & 0.589 & Quercetin \\
\hline 16 & IL1B & 39 & 0.023 & 0.593 & Quercetin, ginsenoside rh2, aloe-emodin \\
\hline
\end{tabular}

(-)-Catechin, quercetin, jaranol, hederagenin, isorhamnetin, beta-sitosterol, 3,9-diO-methylnissolin, 7-O-methylisomucronulatol, 9,10-dimethoxypterocarpan-3-O$\beta$-D-glucoside, (6aR,11aR)-9,10-dimethoxy-6a,11a-dihydro-6H-benzofurano[3,2c]chromen-3-ol, bifendate, formononetin, calycosin, kaempferol, 1,7-dihydroxy3,9-dimethoxy pterocarpene, stigmasterol, aloe-emodin, (2R)-5,7-dihydroxy-2-(4hydroxyphenyl)chroman-4-one, ZINC04073977, mandenol, DFV, EUPATIN, rhein, toralactone, hesperetin, naringenin, 5,7-dihydroxy-2-(3-hydroxy-4methoxyphenyl)chroman-4-one, ginsenoside rh2, 2,6,10,14,18-pentamethylicosa2,6,10,14,18-pentaene, cornudentanone, hydroxygenkwanin, aobiletin, tetrahydroalstonine, marmin

$\begin{array}{lccll}18 & \text { NOS3 } & 27 & 0.018 & 0.569 \\ 19 & \text { CCL2 } & 35 & 0.017 & 0.586 \\ 20 & \text { SERPINE1 } & 20 & 0.016 & 0.527 \\ 21 & \text { MAPK3 } & 33 & 0.015 & 0.582 \\ & & & & \\ 22 & \text { ESR1 } & 25 & 0.014 & 0.544 \\ & & & & \\ 23 & \text { CREB1 } & 24 & 0.014 & 0.541 \\ 24 & \text { IL10 } & 33 & 0.012 & 0.576 \\ 25 & \text { CXCL8 } & 35 & 0.012 & 0.579 \\ 26 & \text { RELA } & 30 & 0.011 & 0.556 \\ 27 & \text { PPARG } & 27 & 0.011 & 0.559\end{array}$

Quercetin, kaempferol, isorhamnetin, 3,9-di-O-methylnissolin

Quercetin

Quercetin

Kaempferol, nobiletin

(-)-Catechin, isorhamnetin, 3,9-di-O-methylnissolin, 7-O-methylisomucronulatol, (6aR,11aR)-9,10-dimethoxy-6a,11a-dihydro-6H-benzofurano[3,2-c]chromen-3-ol, formononetin, calycosin, (2R)-5,7-dihydroxy-2-(4-hydroxyphenyl)chroman-4-one, $\mathrm{DFV}$, toralactone, naringenin, aobiletin

\section{Aobiletin \\ Quercetin \\ Quercetin}

Quercetin, isorhamnetin, kaempferol, naringenin

(-)-Catechin, quercetin, isorhamnetin, 7-O-methylisomucronulatol, formononetin, kaempferol, naringenin, aobiletin, ginsenoside $\mathrm{f} 2$, tetrahydroalstonine upper $50 \%$ cluster terms were selected for gene function analysis based on $P$ value $<0.05$. As the results show, BP enrichment was mainly involved in the following gene function (Figure 6 and Supplementary Table S6): positive regulation of transcription DNA-templated (25), lipopolysaccharidemediated signaling pathway (8), and positive regulation of blood vessel endothelial cell migration (7). In addition, BP enrichment was also involved in cellular response to vascular endothelial growth factor (VEGF) stimulus (6), response to oxidative stress (8), positive regulation of reactive oxygen species (ROS) metabolic process (5), protein kinase $B(\mathrm{PKB})$ signaling (5), activation of MAPK activity (7), and cellular oxidant detoxification (6). And MF enrichment was mainly involved in the following gene functions (Figure 7 and Supplementary Table S7): transcription factor binding (15), growth factor activity (15), and heme binding (3). In addition, it was also involved in MAPK activity (15), protein kinase activity (10), and cysteine-type endopeptidase activity involved in apoptotic process (4). And CC enrichment was mainly 
Top 30 GO terms on biological process

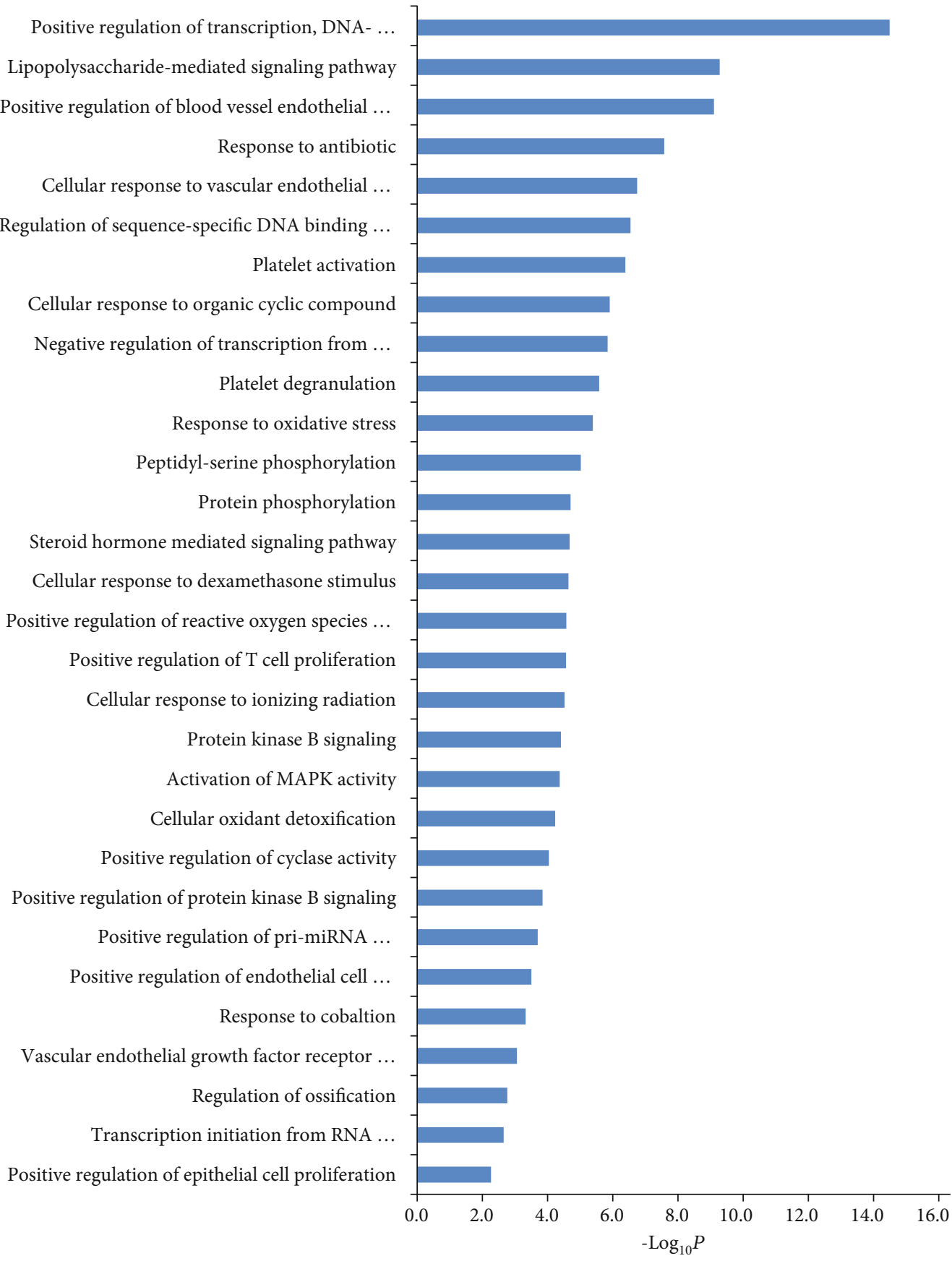

FIgURE 6: The top 30 enriched Gene Ontology (GO) terms for biological process (BP) were analyzed by DAVID system under the condition with $P$ value $<0.05$.

involved in the following gene function (Figure 8 and Supplementary Table S8): external side of plasma membrane (ESM) (10), platelet alpha granule lumen (6), and nuclear chromatin (8). The results showed that gene function involved in many pathological factors of DN such as oxidative stress, inflammatory response, hemodynamic changes, and genetic factors $[7,15,16]$.

3.6. KEGG Pathway Analysis. To further reveal the potential mechanism of the TSF on the effect of DN, we conducted
KEGG pathway enrichment analysis. The 8 annotation clusters were obtained in the KEGG pathway whose enrichment scores were ranged from 2.188 to 12.149 . And the top 30 pathways deeply associated with $\mathrm{DN}$ were screened out based on the $P$ value $<0.001$ (Figure 9 and Supplementary Table S9). Numerous key pathways for cotarget genes were identified, such as the HIF-1 signaling pathway, VEGF signaling pathway, MAPK signaling pathway, TNF signaling pathway, PI3K-Akt signaling pathway, rheumatoid arthritis, mTOR signaling pathway, and 


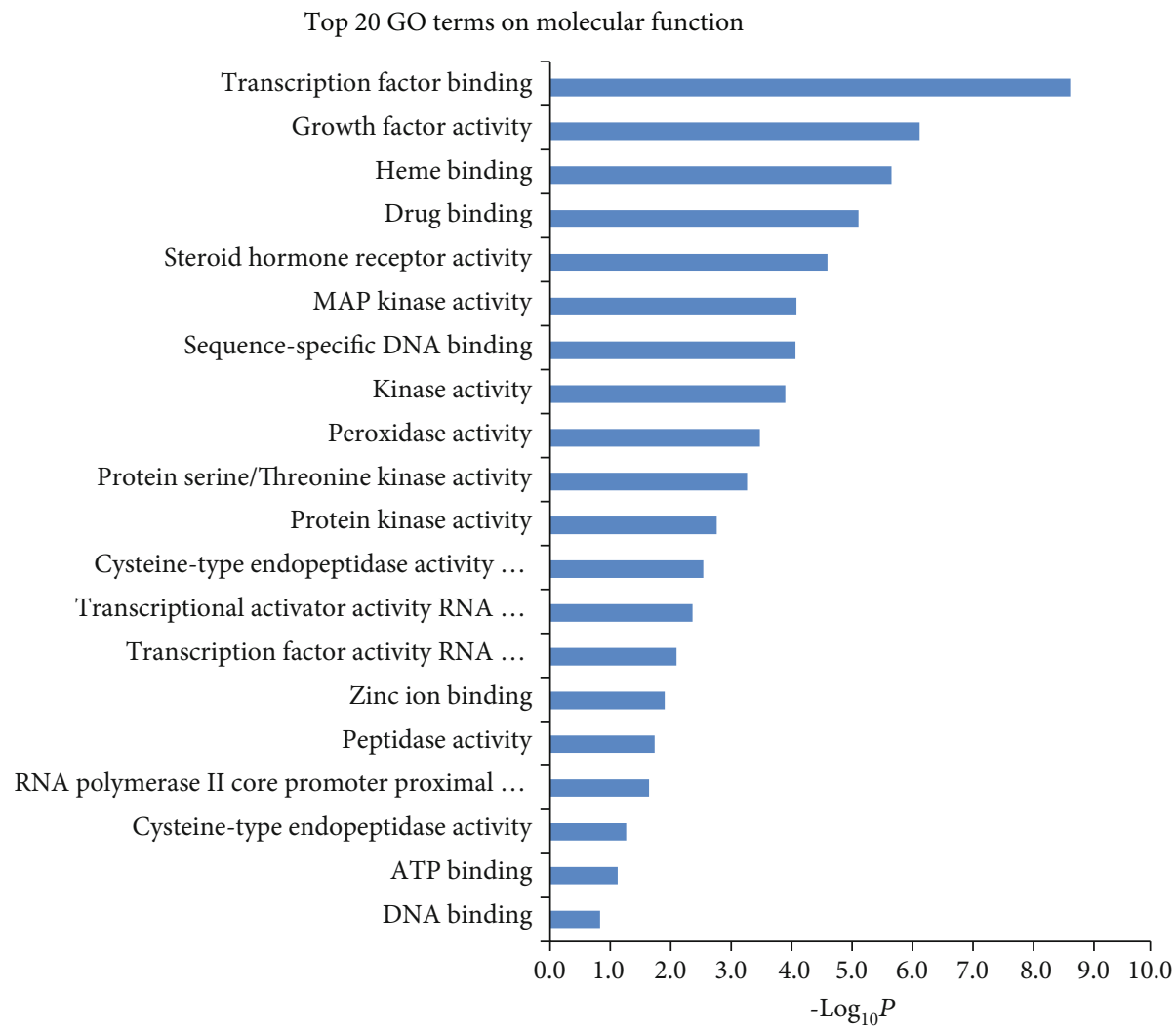

Figure 7: Enriched Gene Ontology (GO) terms for molecular function (MF) were analyzed by DAVID system under the condition with $P$ value $<0.05$.

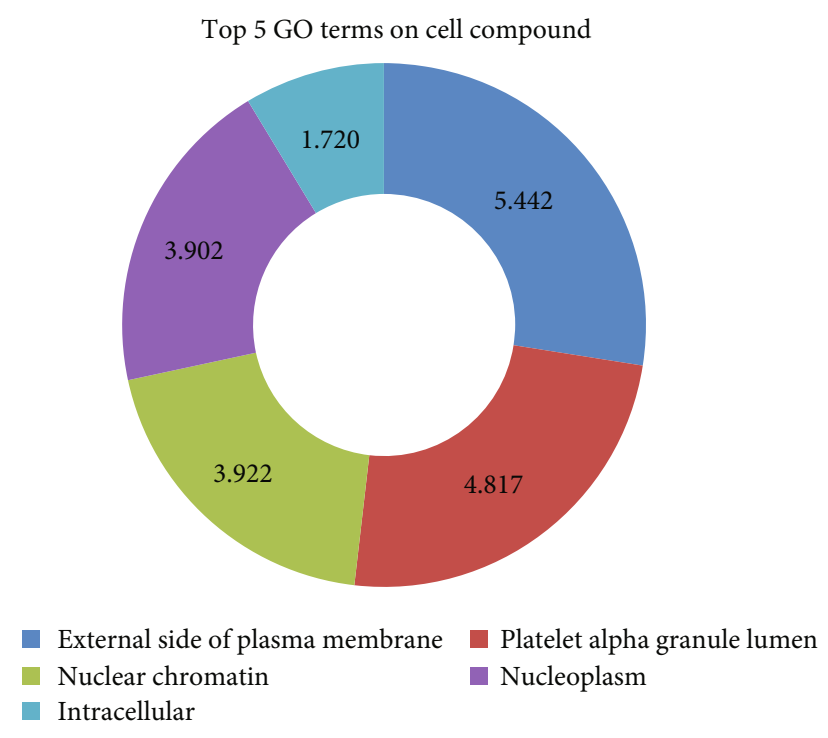

FIgURe 8: Enriched GO terms for the cell compound (CC) were analyzed by the DAVID system under the condition with $P$ value $<0.05$. The value in picture means $-\log _{10} P$.

chemokine signaling pathway related with response to oxidative stress and inflammatory reaction process; insulin resistance, type II diabetes mellitus, and insulin signaling pathway related to the glycometabolism process; nonalcoholic fatty liver disease (NAFLD) and adipocytokine signaling pathway connected with lipid metabolism; Toll-like receptor signaling pathway, NOD-like receptor signaling pathway, $\mathrm{T}$ cell receptor signaling pathway, and focal adhesion linked to immunological stress; and thyroid hormone signaling pathway, renal cell carcinoma, and thyroid cancer associated with renal function. The results suggested that TSF alleviated the DN disease by mainly regulating oxidative stress, inflammatory reaction, glucose and lipid disorders, abnormal renal function, and other pathways.

3.7. Compound-Target-Pathway Network Analysis. In order to further explore the relationship among active compounds, key cotargets, and key pathways, the compound-targetpathway network was constructed by the top 30 pathways with their corresponding cotarget and active compounds (Figure 10 and Supplementary Table S10). According to the compound-target-pathway network, the key active ingredients quercetin, naringenin, kaempferol, and isorhamnetin with three topological parameters higher than the corresponding median values obviously associated with the treatment of $\mathrm{DN}$ by regulating oxidative stress, inhibiting inflammatory reaction, balancing glucose-lipid metabolic process, and improving renal function. Moreover, the PI3K-Akt signaling pathway, TNF signaling pathway, NAFLD, focal adhesion, Rapl signaling pathway, $\mathrm{T}$ cell receptor signaling pathway, MAPK signaling pathway, and insulin resistance with three topological 


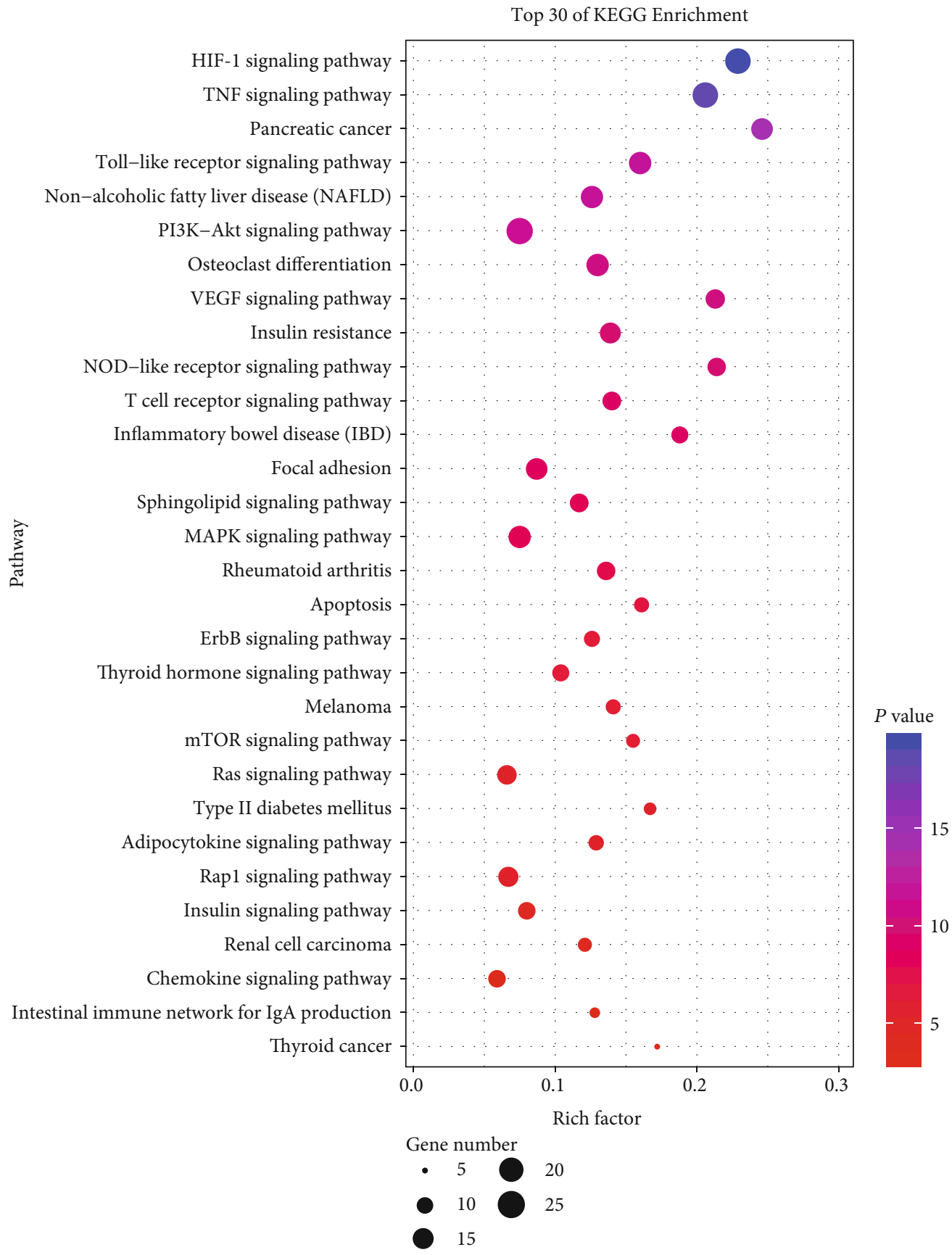

Figure 9: Enriched KEGG terms for TSF on treatment of DN. The $P$ value means $-\log _{10} P$ value, the size of the circle means the gene number, and the color of the circle means the value of $-\log _{10} P$.

parameters higher than the corresponding median values were the major hubs in this network.

\subsection{Determination of the Amounts of Four Key Compounds} in TSF. The established UHPLC-MS/MS analytical method was subsequently applied to successfully determine the quercetin, naringenin, kaempferol, and isorhamnetin (Figure 11) in six TSF samples. And the typical ion chromatograms (TIC) of 4 compounds in the blank sample, standard solution, and sample solution are shown in Figure 12. The average concentrations of quercetin, naringenin, kaempferol, and isorhamnetin were 0.224, 8.295, 0.0564, and $0.0879 \mathrm{mg} \cdot \mathrm{kg}^{-1}$, respectively. The results would provide material basis information for further study on signaling pathways in TSF.

\section{Discussion}

As the single compound medicine is less sufficient for treating complex conditions, multicomponent drugs coupled with multiple targets and synergistic effects on multiple molecular mechanisms are becoming more and more popular for the 


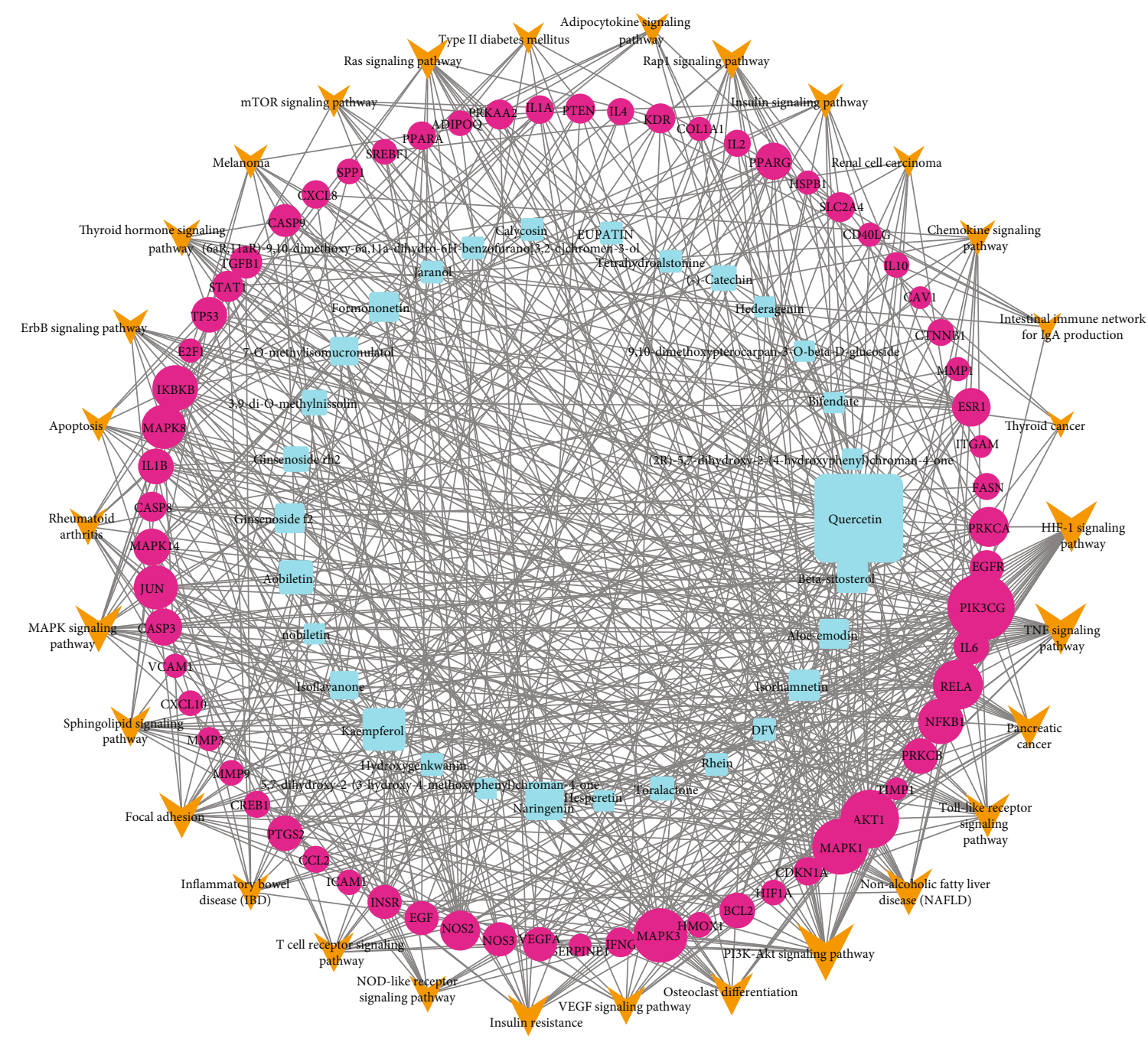

FIGURE 10: The compound-target-pathway network of Tangshen Formula (TSF). The blue square nodes represent compounds. The red circle nodes represent targets. The orange arrows represent pathways. The size of them represent the degree; the lines among inner nodes display the relationship among compounds, targets, and pathways.

treatment of DN. The traditional Chinese medicines as natural medicines show a new source of therapeutic drugs for DN. However, due to a large number of natural compounds in complex herbs, the active ingredients are not clear in the clinical and pharmacological research. In addition, the targets and integral molecular mechanism of multicomponent drugs are not fully illustrated, because the ordinary pharmacological experiments needed consume a large number of resources and time for each compound. Along with the development of bioinformatics technologies, the network pharmacology approach provides an efficient tool to solve the problem of multicomponent, multitarget, and multipathway study in traditional Chinese medicine.

As a traditional Chinese medicine, TSF significantly alleviated proteinuria, enhanced EGFR, and improved phospholipid metabolism for DN patience. However, their active ingredients are not clear in clinical and pharmacological research. In this study, the herb-compound-target network illustrated that the 11 main active compounds including quercetin, kaempferol, ginsenoside f2, 7-O-methylisomucro- nulatol, naringenin, isoflavanone, beta-sitosterol, stigmasterol, formononetin, nobiletin, and isorhamnetin play an important role in TSF. In addition, the protein-protein international network associated with $\mathrm{DN}$ had shown that these key active compounds in TSF were associated with DN targets, too. Thus, it is worth exploring the relationship of these active ingredients with DN. Furthermore, according to the compound-target-pathway network, quercetin, kaempferol, naringenin, and isorhamnetin as the key active ingredients were related to the key molecular mechanisms for TSF treating DN. According to the previous animal research, these active ingredients have been associated with antioxidative stress, inhibiting inflammation, and regulating glucolipid metabolism. Evidence has suggested that quercetin could significantly increase the level of SIRT1, SOD and CAT, decrease NF- $\kappa$ B activity and MDA levels, and suppress oxidative stress in a STZ-induced diabetes rat model [17]; could alleviate renal oxidative stress injury, inhibit inflammatory cell infiltration, and downregulate intercellular adhesion molecular-1 (ICAM-1) expression in diabetic rats [18]; and 
<smiles>O=c1c(O)c(-c2ccc(O)c(O)c2)oc2cc(O)cc(O)c12</smiles>

Quercetin<smiles>O=c1c(O)c(-c2ccc(O)cc2)oc2cc(O)cc(O)c12</smiles>

Kaempferol<smiles>O=C1CC(c2ccc(O)cc2)Oc2cc(O)cc(O)c21</smiles>

Naringenin<smiles>COc1cc(-c2oc3cc(O)cc(O)c3c(=O)c2O)ccc1O</smiles>

Isorhamnetin

FIGURE 11: The chemical structures of quercetin, naringenin, kaempferol, and isorhamnetin.

could improve lipid metabolism by the SCAP-SREBP2-LDLr signaling pathway in type 2 diabetes $\mathrm{db} / \mathrm{db}$ mice with early stage diabetic nephropathy [19]. It is reported that kaempferol could show antifibrosis and anti-inflammation by reducing oxidative stress via the inhibition of the TNF- $\alpha$ p38 MAP kinase signaling pathway in type 2 diabetic mice [20] and could show antifibrotic and renoprotective effects by enhancing GLP-1 and insulin release along with the inhibition of RhoA/Rho kinase in diabetic nephropathy C57BL/6 mice model [21]. The research has indicated that naringenin had anti-inflammatory and antifibrotic effects by decreasing protein kinase $\mathrm{C}$ and restraining NF- $\kappa \mathrm{B}$ p65 activity, mRNA expression, and protein production in kidney tissue of diabetic mice [22] and could ameliorate the renal damage by upregulated PPAR expression, increase CYP4A-20-HETE level in the DN model of a high-fat diet combined with streptozotocin (STZ) [23], and regulate oxidative stress which correlates with decreased cytokine expression and apoptotic events by downregulating the TGF-b1 and IL-1 level for improving ameliorating renal impairment in STZ-induced diabetic rat [24]. It is indicated that isorhamnetin involved renoprotective effects by the NF- $\kappa \mathrm{B}$ signaling pathway in type 2 diabetic rat model constructed by a high-fat diet plus streptozocin injection [25]. In addition, formononetin could increase SIRT1 expression and reduce oxidative stress in kidney tissues of type 2 diabetic rats [26]. And the nobiletin could suppress high-glucose-induced inflammation and ECM accumulation in human mesangial cells via the STAT3/NF- $\kappa$ B pathway [27]. Thus, the relationship of these active ingredients between TSF and DN were indeed worth exploring. Moreover, quercetin, naringenin, kaempferol, and isorhamnetin were successfully detected in TSF by the UHPLC-MS/MS analytical method in this study. And their concentrations were $0.224,8.295,0.0564$, and $0.0879 \mathrm{mg} \cdot \mathrm{kg}^{-}$ ${ }^{1}$, respectively. The material basis results would provide important information for further in vivo and in vitro experimental validations on potential key pharmacological mechanism of TSF on DN.

From protein-protein interaction network analysis, there are 27 key potential targets of TSF associated with DN. And they were further analyzed by Cytoscape software; the PTGS2, PPARG, CASP3, JUN, RELA, MMP9, ESR1, AKT1, NOS3, TP53, HMOX1, MAPK1, IL1B, and MAPK8 might be the hub targets in which TSF could alter the expression of DN-regulated targets and improve DN symptoms. Furthermore, the key targets associated with DN such as PTGS2, PPARG, and RELA were regulated by quercetin, naringenin, kaempferol, and isorhamnetin. In addition, the key target such as IL6, VEGFA, MMP9, EGFR, EGF, CCL2, SERPINE1, IL10, and CXCL8 could be only regulated by quercetin. The results suggested that TSF had the characteristics of multicomponent and multitarget in treating DN.

In order to explore the gene function and pathways of TSF treating on DN, GO enrichment and KEGG pathway enrichment were analyzed. The results indicated that TSF might be related to suppressing oxidative stress, restraining inflammatory reaction, regulating glucose and lipid metabolism disorders, and improving renal function by the HIF-1 signaling pathway, VEGF signaling pathway, MAPK signaling pathway, TNF signaling pathway, PI3K-Akt signaling pathway, mTOR signaling pathway, NOD-like receptor signaling pathway, $\mathrm{T}$ cell receptor signaling pathway, lipopolysaccharide-mediated signaling pathway, insulin signaling pathway, adipocytokine signaling pathway, thyroid hormone signaling pathway, cysteine-type endopeptidase activity involved in apoptotic process, and other pathways. According to the compound-target-pathway network analysis, the PI3K-Akt signaling pathway, TNF signaling pathway, NAFLD, focal adhesion, rap1 signaling pathway, T cell receptor signaling pathway, MAPK signaling pathway, and insulin 

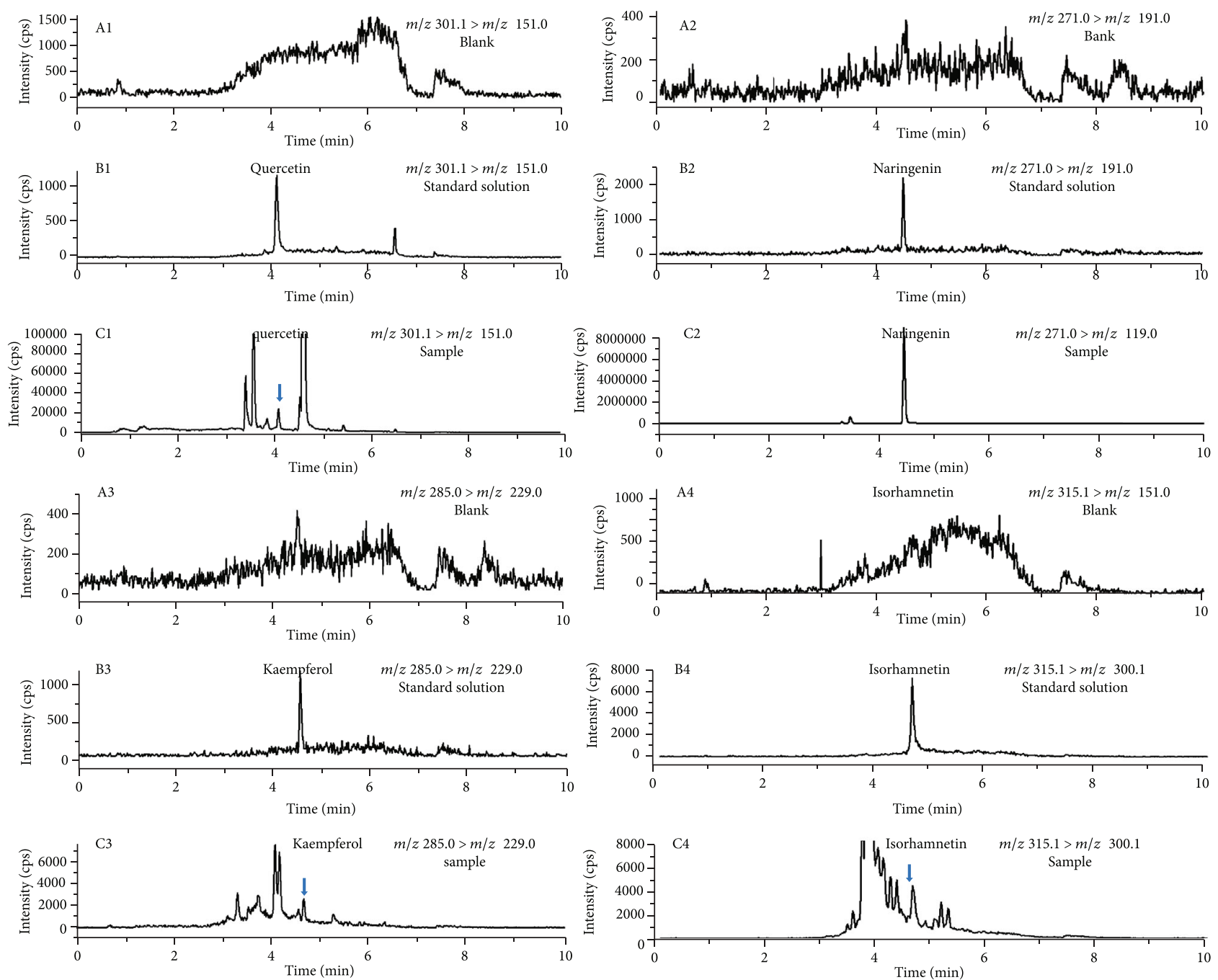

FIGURE 12: The TIC chromatographs of quercetin, naringenin, kaempferol, and isorhamnetin in blank solution (A1-A4), standard solution spiked at limit of quantitation (B1: $0.01 \mathrm{ng} / \mathrm{mL}, \mathrm{B} 2: 0.005 \mathrm{ng} / \mathrm{mL}, \mathrm{B} 3: 0.2 \mathrm{ng} / \mathrm{mL}$, and B4: $0.01 \mathrm{ng} / \mathrm{mL}$ ) and sample solution (C1-C4).

resistance showed the key pathways for TSF in the treatment of DN. As we know, the pathogenesis of DN might be mainly related with inflammatory response, oxidative stress, glucose metabolism disorders, abnormal lipid metabolism, genetic factors, and hemodynamic changes [5]. And the abnormal inflammatory and oxidative stress process plays significant roles in the pathogenesis and development of DN. The previous researches have shown that the PI3K-Akt signaling pathway, TNF signaling pathway, rap1 signaling pathway, and MAPK signaling pathway play crucial roles in inflammatory response and oxidative stress. Shi et al. [5] have alleviated inflammatory response and improved renal function by reducing the level of TGF- $\beta 1$, TNF- $\alpha$, IL6, and IL- $1 \beta$ and inhibiting the PTEN-PI3K-Akt signaling pathway in a DN rat model via STZ. Jiang et al. [28] have reported reducing oxidative stress and inflammation response by regulating the MAPK signaling pathway including attenuating the expression of p38 MAPK, TNF- $\alpha$, COX-2, and MMP9 for protecting against renal injury in $\mathrm{DN}$ rats induced by feeding a high-fat diet and STZ. Xiao et al. have ameliorated oxidative stress and renal tubular injury by the Raplb-C/EBP-b-PGC- 1a signaling pathway in high glucose- (HG-) induced mitochondrial dysfunction in tubular cells [29]. Zhu et al. [30] have inhibited oxidative stress and reduced inflammatory responses by the inhibition of the MAPK signaling pathway for protecting kidney injury in high-fat diet/streptozotocininduced diabetic mice. In addition, the VEGF signaling pathway play an important role in occurrence and development of DN [31], and the HIF-1 signaling pathway could regulate the expression of VEGFA in inflammatory response and prevent kidney tissue damage [32]. Moreover, the PI3K-Akt signaling pathway also had acted on decreasing renal fibrosis and renal impairment. Song et al. [33] have suggested inhibiting the renal interstitial fibrosis by reducing the TGF- $\beta 1$ mediated elevation of Col I, FN, and $\alpha$-SMA through the PTEN/PI3K/Akt pathway in STZ-induced diabetic mice. Ni et al. [34] have ameliorated renal impairment and inhibited podocyte dysfunction by targeting the PI3K/Akt pathway in a DN rat model with a high-glucose/high-lipid diet. Furthermore, NAFLD were significantly related to lipid metabolism, insulin resistance was obviously associated with glucose metabolism, and focal adhesion and the $\mathrm{T}$ cell receptor 
signaling pathway were linked to immunological stress. Therefore, multipathways in TSF had a synergistic effect for treating DN.

In this study, the results further confirm the previous work on TSF. Based on these, the new predicted pathways such as the PI3K-Akt signaling pathway and the MAPK signaling pathway and the key compounds such as quercetin, kaempferol, naringenin, and isorhamnetin were worth further exploring for TSF treating DN in in vivo and in vitro experimental validations.

\section{Conclusions}

In this study, a network pharmacology approach was firstly applied to explore multiple active compounds and multiple key pharmacological mechanisms for TSF treating DN by herb-compound-target network, protein-protein interaction network, and compound-target-pathway network analysis. And the results showed that TSF have the characteristic of multicomponents, multitargets, multipathways, and synergistic effects for treating DN. Quercetin, naringenin, kaempferol, and isorhamnetin as key active compounds and the PI3K-Akt signaling pathway, TNF signaling pathway, NAFLD, focal adhesion, rap1 signaling pathway, T cell receptor signaling pathway, MAPK signaling pathway, and insulin resistance as the key molecular mechanisms play an important role in TSF treating DN. Moreover, quercetin, naringenin, kaempferol, and isorhamnetin were successfully detected in TSF by UHPLC-MS/MS. And their concentrations were $0.224,8.295,0.0564$, and $0.0879 \mathrm{mg} \cdot \mathrm{kg}^{-1}$, respectively. Although the present study provided a methodological exploration and analysis method for identifying key active compounds and potential pharmacological mechanism of TSF on DN, in vivo and in vitro experimental validations are required in the next study to support our findings.

\section{Data Availability}

The tables data used to support the findings of this study are included within the supplementary information files.

\section{Conflicts of Interest}

The authors declare no conflict of interest.

\section{Authors' Contributions}

Conceptualization was handled by W.Z. and P.L.; methodology was handled by W.Z.; software was handled by W.Z.; validation was handled by W.Z., X.F., Y.Z., and W.W.; formal analysis was handled by W.Z., X.Z., and Y.W.; investigation was handled by P.L.; resources were handled by Y.W., H.Z., L.P., T.Z., and H.Z.; data curation was handled by W.Z. and X.Z.; original draft preparation was handled by W.Z.; review and editing was handled by W.Z. and P.L.; visualization was handled by P.L.; project administration was handled by P.L.; and funding acquisition was handled by P.L.

\section{Acknowledgments}

We acknowledge professor Dr. Feng Zhang who supported this research. This research was funded by the National Natural Science Foundation of China, grant number “81620108031.”

\section{Supplementary Materials}

The Supplementary Tables S1-S10 are available online. (Supplementary Materials)

\section{References}

[1] A. Verma, A. B. Patel, A. Upadhyay, and S. S. Waikar, "CREDENCE: significant victory for diabetic kidney disease," Trends in Endocrinology \& Metabolism, vol. 31, no. 6, pp. 391-393, 2020.

[2] N. P. Marketou, G. P. Chrousos, and C. K. Gantenbein, "Diabetic nephropathy in type 1 diabetes: a review of early natural history, pathogenesis and diagnosis," Diabetes/Metabolism Research and Reviews, vol. 33, no. 2, article e2841, 2017.

[3] G. D. Sun, C. Y. Li, W. P. Cui et al., "Review of herbal traditional Chinese medicine for the treatment of diabetic nephropathy," Journal of Diabetes Research, vol. 2016, Article ID 5749857, 18 pages, 2016.

[4] G. Leoncini, F. Viazzi, S. De Cosmo, G. Russo, P. Fioretto, and R. Pontremoli, "Blood pressure reduction and RAAS inhibition in diabetic kidney disease: therapeutic potentials and limitations," Journal of Nephrology, vol. 3, pp. 949-963, 2020.

[5] C. H. Shi, Y. Huang, W. Q. Li, and R. G. Chen, "Influence of LncRNA UCA1 on glucose metabolism in rats with diabetic nephropathy through PI3K-Akt signaling pathway," European Review for Medical and Pharmacological Sciences, vol. 23, no. 22, pp. 10058-10064, 2019.

[6] G. C. W. Chan and S. C. W. Tang, "Diabetic nephropathy: landmark clinical trials and tribulations," Nephrology Dialysis Transplantation, vol. 31, no. 3, pp. 359-368, 2016.

[7] J. Barrera-Chimal and F. Jaisser, "Pathophysiologic mechanisms in diabetic kidney disease: a focus on current and future therapeutic targets," Diabetes, Obesity and Metabolism, vol. 22, no. S1, pp. 16-31, 2020.

[8] D. Dragoș, M. M. Manea, D. Timofte, and D. Ionescu, "Mechanisms of herbal nephroprotection in diabetes mellitus," Journal of Diabetes Research, vol. 2020, Article ID 5710513, 31 pages, 2020.

[9] P. Li, Y. P. Chen, J. P. Liu et al., "Efficacy and safety of Tangshen formula on patients with type 2 diabetic kidney disease: a multicenter double-blinded randomized placebo-controlled trial," PloS One, vol. 10, no. 5, article e0126027, 2015.

[10] X. Yang, B. X. Zhang, X. G. Lu et al., "Effects of Tangshen formula on urinary and plasma liver-type fatty acid binding protein levels in patients with type 2 diabetic kidney disease: posthoc findings from a multi-center, randomized, double-blind, placebo-controlled trial investigating the efficacy and safety of Tangshen formula in patients with type 2 diabetic kidney disease," BMC Complementary and Alternative Medicine, vol. 16, p. 246, 2016.

[11] M. Huang, C. Zhu, Q. L. Liang et al., "Effect of Tangshen formula on phospholipids metabolism in diabetic nephropathy patients," Acta Pharmaceutica Sinica, vol. 46, no. 7, pp. 780786, 2011. 
[12] Q. Kong, H. Zhang, T. Zhao et al., "Tangshen formula attenuates hepatic steatosis by inhibiting hepatic lipogenesis and augmenting fatty acid oxidation in $\mathrm{db} / \mathrm{db}$ mice," International Journal of Molecular Medicine, vol. 38, no. 6, pp. 1715-1726, 2016.

[13] T. T. Zhao, S. F. Sun, H. J. Zhang et al., "Therapeutic effects of Tangshen formula on diabetic nephropathy in rats," PLoS One, vol. 11, no. 1, article e0147693, 2016.

[14] A. L. Hopkins, "Network pharmacology: the next paradigm in drug discovery," Nature Chemical Biology, vol. 4, no. 11, pp. 682-690, 2008.

[15] E. A. T. Koch, R. Nakhoul, F. Nakhoul, and N. Nakhoul, "Autophagy in diabetic nephropathy: a review," International Urology and Nephrology, vol. 52, pp. 1705-1712, 2020.

[16] L. Zhou, W. Huang, Y. Xu et al., "Sweet taste receptors mediated ROS-NLRP3 inflammasome signaling activation: implications for diabetic nephropathy," Journal of Diabetes Research, vol. 2018, Article ID 7078214, 15 pages, 2018.

[17] H. Iskender, E. Dokumacioglu, T. M. Sen, I. Ince, Y. Kanbay, and S. Saral, "The effect of hesperidin and quercetin on oxidative stress, NF- $\kappa$ B and SIRT1 levels in a STZ-induced experimental diabetes model," Biomedicine \& Pharmacotherapy, vol. 90, pp. 500-508, 2017.

[18] F. Tong, S. H. Liu, B. Yan, X. F. Li, S. W. Ruan, and S. Y. Yang, "Quercetin nanoparticle complex attenuated diabetic nephropathy via regulating the expression level of ICAM-1 on endothelium," International Journal of Nanomedicine, vol. Volume 12, pp. 7799-7813, 2017.

[19] X. Jiang, J. Yu, X. Wang, J. Ge, and N. Li, “Quercetin improves lipid metabolism via SCAP-SREBP2-LDLr signaling pathway in early stage diabetic nephropathy," Diabetes, Metabolic Syndrome and Obesity: Targets and Therapy, vol. 12, pp. 827-839, 2019.

[20] H. Y. Wang, J. G. Zhao, Z. G. Wei, and Y. Q. Zhang, "The renal protection of flavonoid-rich ethanolic extract from silkworm green cocoon involves in inhibiting TNF- $\alpha$-p38 MAP kinase signalling pathway in type 2 diabetic mice," Biomedicine \& Pharmacotherapy, vol. 118, p. 109379, 2019.

[21] D. Sharma, R. K. Tekade, and K. Kalia, "Kaempferol in ameliorating diabetes-induced fibrosis and renal damage: an in vitro and in vivo study in diabetic nephropathy mice model," Phytomedicine, vol. 76, p. 153235, 2020.

[22] S. J. Tsai, C. S. Huang, M. C. Mong, W. Y. Huang, H. Y. Kam, and M. C. Yin, "Anti-inflammatory and antifibrotic effects of naringenin in diabetic mice," Journal of Agricultural and Food Chemistry, vol. 60, pp. 514-521, 2011.

[23] S. Ding, H. Qiu, J. Huang et al., “Activation of 20-HETE/PPARs involved in reno-therapeutic effect of naringenin on diabetic nephropathy," Chemico-Biological Interactions, vol. 307, pp. 116-124, 2019.

[24] S. Roy, F. Ahmed, S. Banerjee, and U. Saha, "Naringenin ameliorates streptozotocin-induced diabetic rat renal impairment by downregulation of TGF- $\beta 1$ and IL- 1 via modulation of oxidative stress correlates with decreased apoptotic events," Pharmaceutical Biology, vol. 54, no. 9, pp. 1616-1627, 2016.

[25] S. Qiu, G. Sun, Y. Zhang, X. L. Li, and R. Wang, "Involvement of the NF- $\kappa$ B signaling pathway in the renoprotective effects of isorhamnetin in a type 2 diabetic rat model," Biomedical Reports, vol. 4, no. 5, pp. 628-634, 2016.
[26] M. J. Oza and Y. A. Kulkarni, "Formononetin attenuates kidney damage in type 2 diabetic rats," Life Sciences, vol. 219, pp. 109-121, 2019.

[27] Z. Liu, Y. Han, F. Zhao, Z. Zhao, J. Tian, and K. Jia, "Nobiletin suppresses high-glucose-induced inflammation and ECM accumulation in human mesangial cells through STAT3/NF$\kappa \mathrm{B}$ pathway," Journal of Cellular Biochemistry, vol. 120, no. 3, pp. 3467-3473, 2018.

[28] Y. Jiang, J. Liu, Z. Zhou, K. Liu, and C. Liu, "Fangchinoline protects against renal injury in diabetic nephropathy by modulating the MAPK signaling pathway," Experimental and Clinical Endocrinology \& Diabetes, vol. 128, no. 8, pp. 499-505, 2020.

[29] L. Xiao, X. Y. Zhu, S. K. Yang et al., "Rap1 ameliorates renal tubular injury in diabetic nephropathy," Diabetes, vol. 63, no. 4, pp. 1366-1380, 2014.

[30] Y. Zhu, C. Zhu, H. Yang, J. J. Deng, and D. D. Fan, “Protective effect of ginsenoside Rg5 against kidney injury via inhibition of NLRP3 inflammasome activation and the MAPK signaling pathway in high-fat diet/streptozotocin-induced diabetic mice," Pharmacological Research, vol. 155, p. 104746, 2020.

[31] T. Nakagawa, "Uncoupling of the VEGF-endothelial nitric oxide axis in diabetic nephropathy: an explanation for the paradoxical effects of VEGF in renal disease," American Journal of Physiology-Renal Physiology, vol. 292, no. 6, pp. F1665-F1672, 2007.

[32] R. Bohuslavova, R. Cerychova, K. Nepomucka, and G. Pavlinkova, "Renal injury is accelerated by global hypoxia-inducible factor 1 alpha deficiency in a mouse model of STZ-induced diabetes," BMC Endocrine Disorders, vol. 17, no. 1, pp. 1-12, 2017.

[33] Y. Song, W. Liu, K. Tang, J. T. Zang, D. Li, and H. Gao, “Mangiferin alleviates renal interstitial fibrosis in streptozotocininduced diabetic mice through regulating the PTEN/PI3K/Akt signaling pathway," Journal of Diabetes Research, vol. 2020, Article ID 9481720, 12 pages, 2020.

[34] W. J. Ni, H. Zhou, H. H. Ding, and L. Q. Tang, "Berberine ameliorates renal impairment and inhibits podocyte dysfunction by targeting the phosphatidylinositol 3-kinase-protein kinase B pathway in diabetic rats," Journal of Diabetes Investigation, vol. 11, no. 2, pp. 297-306, 2019. 